\title{
Challenges in network science: Applications to infrastructures, climate, social systems and economics
}

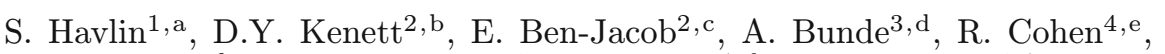

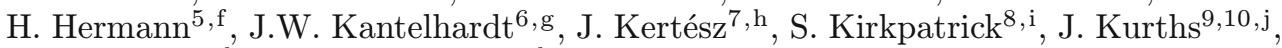
J. Portugali ${ }^{11, k}$, and S. Solomon ${ }^{12,1}$

1 Minerva Center and Department of Physics, Bar-Ilan University, Ramat-Gan 52900, Israel

2 School of Physics and Astronomy, Tel-Aviv University, Tel Aviv 69978, Israel

3 Institut für Theoretische Physik III, Justus-Liebig-Universität Giessen, 35392 Giessen, Germany

4 Department of Mathematics, Bar-Ilan University, Ramat-Gan 52900, Israel

5 Computational Physics for Engineering Materials, IfB, ETH Zurich, Schafmattstrasse 6, 8093 Zurich, Switzerland

${ }^{6}$ Institut für Physik, Martin-Luther-Universität Halle-Wittenberg, 06099 Halle, Germany

7 Institute of Physics, Budapest University of Technology and Economics,

1111 Budapest, Hungary

8 School of Computer Science and Engineering, The Hebrew University of Jerusalem, Jerusalem 91904, Israel

9 Institute of Physics, Humboldt University Berlin, 12489 Berlin, Germany

10 Potsdam Institute for Climate Impact Research, 14412 Potsdam, Germany

11 Department of Geography, Tel-Aviv University, Ramat Aviv, Tel Aviv 69978, Israel

12 Racah Institute of Physics, The Hebrew University of Jerusalem, Jerusalem 91904, Israel

Received 1 August 2012 / Received in final form 9 October 2012

Published online 5 December 2012

\begin{abstract}
Network theory has become one of the most visible theoretical frameworks that can be applied to the description, analysis, understanding, design and repair of multi-level complex systems. Complex networks occur everywhere, in man-made and human social systems, in organic and inorganic matter, from nano to macro scales, and
\end{abstract}

\footnotetext{
${ }^{a}$ e-mail: havlins@gmail.com

b e-mail: drorkenett@gmail.com

c e-mail: eshelbj@gmail.com

d e-mail: armin. bunde@physik.uni-giessen.de

e e-mail: reuven@macs.biu.ac.il

f e-mail: hans@ifb.baug.ethz.ch

g e-mail: jan.kantelhardt@physik.uni-halle.de

h e-mail: kertesz@phy . bme.hu

i e-mail: kirk@cs.huji.ac.il

j e-mail: kurths@pik-potsdam.de

k e-mail: juval@post.tau.ac.il

${ }^{1}$ e-mail: sorin@vms.huji.ac.il
} 
in natural and anthropogenic structures. New applications are developed at an ever-increasing rate and the promise for future growth is high, since increasingly we interact with one another within these vital and complex environments. Despite all the great successes of this field, crucial aspects of multi-level complex systems have been largely ignored. Important challenges of network science are to take into account many of these missing realistic features such as strong coupling between networks (networks are not isolated), the dynamics of networks (networks are not static), interrelationships between structure, dynamics and function of networks, interdependencies in given networks (and other classes of links, including different signs of interactions), and spatial properties (including geographical aspects) of networks. This aim of this paper is to introduce and discuss the challenges that future network science needs to address, and how different disciplines will be accordingly affected.

\section{Introduction and overview}

Within the span of a decade, network theory has become one of the most visible theoretical frameworks that can be applied to the description, analysis, understanding, design and repair of complex systems and in particular in strongly coupled multi-level complex systems. Complex networks occur everywhere, in man-made systems and in human social systems, in organic and non-organic matter from nano to macro scales, and in natural and anthropogenic structures. Examples include linked molecular or cellular structures, climate networks, communication and infrastructure networks, but also social and economic networks. An understanding of the growth, structure, dynamics, and functioning of these networks and their mutual interrelationships is essential in order to find precursors of changes, to make the systems resilient against failures, to protect them against external attacks or, as in the case of terroristic networks and misleading social manipulation strategies, to be able to fight them in the most efficient way, while supporting objective public information and opinion formation. The interrelationship between structure (topology) and dynamics, function and task performance in complex systems represents the focus of many studies in different fields of research with important scientific and technological applications. Because of their enormous potential to represent the intricate topology of numerous systems in nature, complex networks have recently been used as a framework to describe the behavior of physical, chemical, biological, technological and social networks. As such, and taking into account the multitude of disciplines in which network science is needed, such research requires intimate interdisciplinary cooperation between computer scientists, social scientists, engineers, mathematicians, and physicists. Such a synergy is the core basis for the FuturICT Flagship project [1-5], which aims to make use of big data to understand, crate and manage complex techno-socio-economic systems $[6,7]$.

Network science has greatly evolved in the past decade and a half, and is currently a leading scientific field in the description of complex systems, which affects every aspect of our daily life [8-11]. Famous examples include the findings about sexual partners [12], Internet and WWW [13,14], epidemic spreading [15], immunization strategies [16], citation networks [17], structure of financial markets [18], social percolation and opinion dynamics [19-21], structure of mobile communication network [23], and many others. Among the phenomena that have been shown to fall in this conceptual framework are: cascading failures, blackouts, crashes, bubbles, crises, viral attacks and defense against them, introduction of new technologies, infrastructure, 
understanding measuring and predicting the emergence and evolution of networks and their stylized features, spreading phenomena and immunization strategies, as well as the stability and fragility of airline networks [11].

Current and past research has shown that in real life systems, there is a strong feedback between the micro states and macro states of the system. This description of nature can be well represented by network science - in which the micro is represented by the nodes of the network and the links between them, and the macro by the network itself, its topology, dynamics and function. Thus, network science, present and future, is the leading framework to investigate real life systems. For example, as opposed to physical systems where the dynamics is usually bottom-up, in social and economic systems there are interplays on all levels with singular top-down feedbacks. Thus, in many practical realizations, in addition to the bottom-up contagion propagation mechanisms one finds that there is a global-to-local feedback: individuals, their interdependence and behaviors build up the system that finally affects back on individuals' choices. It has been proposed that the bottom/up - top/down feedback has the capability to change completely the character of a phase transition from continuous to discontinuous, thus explaining the severity of the economic crises in systems where the collective interacts as such with its own components [24] (see also box 5 of [25]).

In past research of network science, investigations of the elementary mechanisms for generating and evolving networks was limited to building models that reproduce stylized facts of natural networks. Over the last few years, it has become possible to empirically observe the elementary moves responsible for network changes at the node and link level. Furthermore, empirical investigations have been aimed at understanding how real networks grow: the appearance of new links preferentially between nodes [26]. In order to connect the macroscopic properties of networks to the elementary node-link dynamics, it is of crucial importance to understand the detailed causal order in which the microscopic dynamics take place. Despite all the great successes of this field [27], the developments have been limited. This aspect of co-evolution of the network with the dynamics on it has only been recently touched upon [28-30]. This is mainly due to the fact that so far crucial aspects have been ignored. For example, it is usually the assumption that links are constant and stable, whereas in real life this is not the case - people change their friends, and their interactions - and this fact is highly missing from the formalism of the classical theories. In addition, the detailed investigation of processes such as spreading (e.g. [31]) reveals how the dynamics on different scales influence the global behavior. These studies have to be carried out in a multi-scale fashion, starting from the inhomogeneous bursting behavior at the individual level [32], to the mesoscopic level, community structures, and the network as a whole.

Until now, there has been a significant advance in understanding the structure and function of networks, and mathematical models of networks are now widely used to describe a broad range of complex systems, from techno-social systems to interactions amongst proteins [33]. However, current methods deal almost exclusively with individual networks treated as isolated systems. In reality an individual network is often just one component in a much larger complex multi-level network (network of networks). As technology has advanced, the coupling is becoming stronger and stronger. For example, there is a strong coupling between human mobility (which can be tracked by mobile networks) and transport networks. In these interdependent networks, the failures of nodes in one network will cause failures of dependent nodes in other networks, and vice-versa [34]. This process happens recursively, and leads to a cascade of failures in the network of networks system. As in physics, when only the individual particles were studied it was made possible to understand the properties of 
gas; however, when the transition was made to study the interactions between these particles, it was finally made possible to understand and describe liquids and solids. Thus, such a transition in network science will lead to a significant paradigm shift, which will reveal a multitude of new features and phenomena.

Spatial constraints create, for example, clustering in the network, affecting the topology of the network and as a consequence the function of the network - unlike the classical random graph theory in which spatial constraints are ignored. The introduction of the methodological framework of small world networks [35] was the first step in this direction, but most real networks in nature are not in fact small world networks. In a small world network every pair of nodes has the same probability to be connected, but in most real networks this probability decays with the distance between the nodes $[36,37]$. It is important to note that the geographic viewpoint is essential even in networks, which are not a priori spatial. An example is the mobile phone cell network, where the so-called gravity law [38] relates the intensity of the relationships to the spatial distance.

The aim of this paper is to review many of the challenges that the current network science faces, and to present future directions and applications which will shape the next generation of network science. The traditional formalism mostly did not take into account many realistic features, such as: coupling between networks (assuming the networks are isolated), the dynamics of networks (assuming the networks are static), interrelationships between structure, dynamics and function of networks, interdependencies in given networks (and other classes of links), co-evolution of networks, and spatial properties of the networks. Answering questions raised with these issues will lead to a new generation of network science, which will provide us with new tools to understand the world we live in, to better design, predict, and defend many aspects of our social life.

\section{State of the art}

Network theory has revolutionized our understanding of complex systems in diverse areas and offers a deeper understanding on how e.g., people, computers, or proteins are connected among their kind. Many systems can be efficiently modeled using a network structure where the system entities are the network nodes and the relations between the entities are the network links [8-11,14,35,49-57]. The universal appeal of the field led researchers from different disciplines to embrace network theory as a common paradigm of true inter-discipliner nature. The intense research on network science has established itself as a new research field with its distinct set of challenges and accomplishments. Many important applications have been developed, such as the design of robust networks or efficient immunization strategies, based mainly on statistical physics approaches. The field is currently extremely active and new interesting challenges arise constantly, with applications in complex social, economic, climate, technological, and biological systems.

One well known example of networks are the different types of infrastructure networks that our daily lives depend on. Most infrastructure networks are used as means of transporting goods, energy or information [58-60]. Information flows through communication networks, epidemics, rumors and opinions through social networks, electrical power through the power grid [61-63] and land transportation through road and railway networks [64], etc. Although there is considerable interplay between the structure of these networks and their transport properties, such as capacity, bottleneck structure and delay, only few studies have focussed on the relations between topology and transport properties [65]. 
The topology of complex real-world networks has a direct impact on their stability when changes in the interaction (link) strengths or failures of nodes occur. The systems may thus be under constant risk with respect to random and targeted attacks on their components $[16,50,66-68]$. A small fraction of such changes may promote a cascading dynamics that spreads through the system affecting a large fraction of its elements $[15,42,52,69-77]$. Thus, much work is being devoted to define suitable measures to quantify the system resilience [42] and to identify the mechanisms/processes responsible for these vulnerabilities [78,79]. Such knowledge will make it possible to develop strategies to optimize existing networks as well as to design resilient ones from scratch $[41,42,80-84]$.

Very recently, it has been shown that the risk to fail drastically increases due to the coupling between systems $[34,85]$. The relevant infrastructures for daily life (oil, transportation, electric power, natural gas, water, and communications) are interdependent and failures taking place in one are very likely to affect the others, leading to large scale phenomena like, e.g., the 2003 blackout affecting Italy and Switzerland [86]. Examples can also be found in economy in the role of the interplay between banks, companies, and countries in the recent financial crises [87]. Unlike single networks, interdependent networks behave very differently [34,88]. Moreover, network theory has focused on networks containing either connectivity links [50,67,89-94] or dependency links [69,71,95-97]. A first mathematical framework to study the robustness of a system when both type of links are present was developed only recently [98]. While connectivity links enable nodes to function cooperatively as a network and a node can function as long as it is connected to the majority of the network, dependency links represent local relations in the sense that, when a node fails, its direct dependency neighbors also fail, independent of the structure of the network. By investigating structural properties of coupled networks, several features of systems have been recently uncovered [83,98-100].

As mentioned above, the main focus of research in network science has been on static networks. Static networks refer to such networks constructed for time series of given variables, for a chosen time horizon. Very recent work, however, has shown that in many real networks, known as temporal networks [102], links are generated, disappear and reappear over time. In traffic communication via ad-hoc networks between vehicles, for example, the presence or absence of links changes very quickly, even on time scales comparable with those for information flow [103]. A co-evolving network, where the function of the systems influences its topology, has only been started to be investigated [28-30]. Furthermore, when modeling the structure (topology) and function of complex networks, it is usually assumed that spatial constraints can be neglected. For a large fraction of real networks, however, a few recent papers suggest that the embedding in $2 d$ or $3 d$ space matters and the geometrical distance between connected nodes plays an important role [36,37,104]. Regarding traffic communication via ad-hoc networks between vehicles, even an embedding in quasi- $1 d$ space is needed because roads are essentially $1 d$ (besides crossings) [64,103].

Network science has been used in many disciplines throughout the year, such as transportation and communication networks. With the outburst of available data, network science has become an essential part of many new areas. For example, the application of complex network theory in climate science is a very young field, where only few studies have been published [48,105-111]. More importantly, it is now possible to quantitatively investigate social networks and social interactions. Social networks dominate social interaction - be they friend networks, mobile phone networks, Internet based (Facebook or Twitter), and others. These networks are playing a significant role in shaping social reality. Network concepts have been proven very useful for the analysis and understanding of scientific collaborations $[112,113]$ or organizational laws of social interactions, such as friendships $[114,115]$. 


\section{Opportunities}

A revolution in network science will provide a wealth of new understanding on our daily life, and will greatly impact it. Many scientific advances will result from such a revolution, which will significantly alter our understanding of social infrastructure and organization, as well as natural multi-level complex systems, i.e. climate. Below we present a partial list of applications that advanced network science is expected to bring forth.

\section{Better immunization strategies}

All of the networks we depend on for our daily lives are vulnerable to attacks. As we rely on more and more networks, of various types, there is a need to develop new immunization strategies on these coupled networks. One main example is the immunization of mobile phone network, which are becoming widely susceptible to attacks and viruses $[39,40]$. A different example is the need to protect ourselves from contagions and viruses spread through air travel [41].

2. Improve robustness of networks in crises and disasters

Critical networks must function in time of crises or disaster. The many disasters that took place in the past decade are usually characterized by a fast collapse of the mobile phone network. This happens when it is most needed to disperse information. Thus, infrastructure networks must be redesigned to be robust [42]. Furthermore, rethinking network topology can greatly improve the transportation of people at time of disasters (as in the recent disaster in Japan, March 2011). A further related aspect is to redesign the processes on networks. Complex systems are per definition out of equilibrium and often in the state or at the edge of instability. The lack of negative feedback loops causes critical response to minor perturbations, which may lead to catastrophic crises. A fundamentally new management of the complexity, which is based on negative feedback loops, will improve the situation. For example, this approach is expected to be crucial for financial systems.

\section{Design of robust and efficient infrastructure}

Stable and resilient infrastructure is critical to our sustainability. New insights from an advanced network science will have a great impact in this regard. Existing infrastructure systems would be made more efficient and resilient, with low costs, and new infrastructure will be designed to this end.

4. Early identification of financial crises

The world is still trying to recover from the 2008 financial crises. These crisis began in the US, and rapidly spread throughout the world; see box 6 of [25] for details. This spreading of the crises, inside and outside of the US, can only be understood when the system is investigated as a set of coupled networks, and their interdependencies. Understanding these couplings and dependencies will play a major role in the prevention of future international crises [43,44].

\section{Blackout avoidance}

Blackouts and power shortages directly result from a cascade of failures, which usually involves several networks - understanding the interactions between networks will lead to knowledge how to prevent these cascades [34]. Furthermore, this understanding will provide real-time online strategies of action during such cascades, before the complete collapse.

6. Early detection of community and social changes

Real life networks are made up of communities and social groups, which are formed on different principles. Changes of these communities result in topological changes of the network, which commonly result in significant changes in the function of the network. By identifying community leaders [45], it will be possible to observe early signs of opinion dynamics and spread of information in different societies. 
Some examples include the recent uprising in Arab countries in the Middle East (during 2011). In order to be able to understand the individuals' different roles in the social networks we need to monitor and categorize typical behavioral patterns.

7. Towards design principles for optimal transport networks

Transportation is a critical element of social interaction. However, past models and design of integrative transportation infrastructures are obsolete. Developing new tools in network science will provide the means to construct more efficient and robust transportation infrastructures (see for example [46,47]).

8. Early detection of extreme climate events

Using networks to represent the strongly coupled components of the climate system provides new and important information on the prediction and understanding of the world's climate. For example, using network theory to describe climate has been shown to provide new robust information on the El-Nino phenomenon $[27,48]$.

\section{Goals}

The main goal of the research proposed here is to develop new approaches and tools in network science based on current and future available data. Reaching this goal will revolutionize our understanding of multi-level complex systems in diverse areas, and will lead to deeper insights on how people, computers or companies are intra and inter connected. Reaching these goals will enable identifying precursors of changes and developing new approaches for the design of integrative systems that are more stable, easier to be protected and repaired and that can perform a wider range of tasks with elevated efficiency. Moreover, network science will pave the way to the development of new classes of self-organized, self-learning and self-improving complex systems. The new tools to achieve this most challenging goal will be based on approaches from statistical physics and computer science: percolation, synchronization, development of new models, many-body interaction, scaling approaches, generating functions, graph theory, methods and algorithms to identify the central and key nodes in the network, spread of information, etc. These methods and algorithms will enable to identify central key players in the network, design robust infrastructure, improve robustness and function of existing infrastructure, revolutionize immunization strategies, understand the coupling and interaction between networks, predict emerging changes and (phase) transitions, prevent cascading failures, as well as developing new understanding on the dynamics, function and task performance of social networks - to name just a few.

\section{Challenges}

Many critical challenges exist, which were not addressed by the early formalization of network science. In this section we discuss the main challenges, whose solution will bring forward a more complete network science, bettering our daily life in all many aspects. We discuss how to develop new frameworks in network science that take into account the important realistic features that have been neglected so far.

\subsection{Coupled multi-level networks (Network of networks)}

The case of a single network that is isolated and does not interact with or depend on other systems rarely occur, just as non-interacting systems in statistical physics. In reality, most network systems continuously interact with other networks, especially 


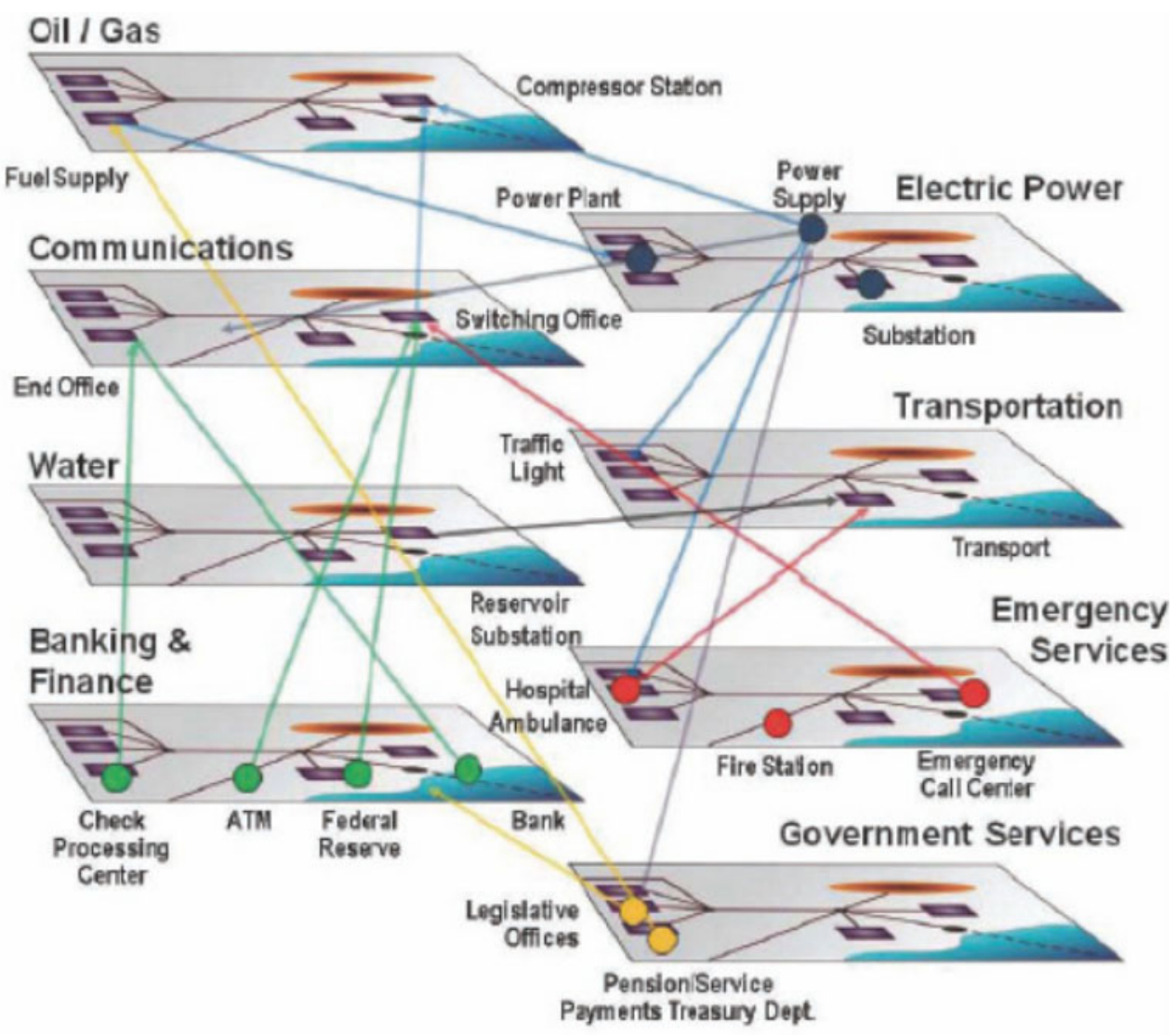

Fig. 1. A coupled and interdependent multi-level network (network of networks). This is an example of how different layers of social organization and infrastructure are represented by networks that are coupled to each other, creating a network of networks.

since modern technology has increased the dependency between networks. Only few and preliminary studies have attempted very recently to face questions relevant to such systems, but they have mainly been qualitative and focused on specific examples. While researchers are beginning to recognize the critical importance of coupling between networks, there is currently no theory or systematic approach for interconnected network systems. This critical void was summarized in a recent US Scientific Congressional Report: 'No currently available modeling and simulation tools exist that can adequately address the consequences of disruptions and failures occurring simultaneously in different critical infrastructures that are dynamically interdependent.' [116]. Understanding the interconnections of networks and their effect on the structural and functional behavior of the coupled system is crucial for properly modeling many real world systems. Introducing coupling between networks is analogous to the introduction of interactions between particles in statistical physics, which led to new cooperative behavior with rich phenomena such as phase transitions. Surprisingly, preliminary results on mathematical models $[34,88]$ show that analyzing complex systems as a network of coupled networks may alter the most basic assumptions that network theory has relied on for single networks. Thus, we aim to generalize the current understanding of network science, developed for individual networks, to 
network of networks. We also aim at modeling and analyzing the robustness of real life network of networks. There are many real life implications to this new conceptual leap in network science, ranging from revolutionary design of infrastructure, organization of the Internet, defense against attacks and viruses, and the stabilization of the world's economies.

\subsection{Dependency relations in networks and networks of networks}

Many systems can be efficiently modeled using a network structure where the system entities are the network nodes and the relations between the entities are the network links (see state-of-the-art). However, many systems are also characterized by small subgroups in which the entities belonging to a group strongly depend on each other. For example, consider a financial network: each company has trading and sales connections with other companies (connectivity links). These connections enable the companies to interact with others and function together as a global financial market. In addition, companies that belong to the same owner strongly depend on one another (dependency links). If one company fails, the owner might not be able to finance the other companies, which will fail too. Another example is an online social network (Facebook or Twitter). Each individual communicates with his friends (connectivity links), thus forming a social network through which information and rumors can spread. However, many individuals will only participate in a social network if other individuals with common interests also participate in that social network, thereby forming dependency groups [98, 117].

Recently, [118] a new approach has been introduced to compute and investigate the mutual dependencies between network nodes from the matrices of node-node correlations. The basis of this methodology is to investigate the partial correlations between a given set of variables (or nodes) of the network. Using the concept of partial correlations [118-124], preliminary results show that it is possible to quantify how one node in the network affects the link between other nodes in the network. This new class of correlation-based networks is able to uncover important hidden information about the system. This methodology is a significant step forward towards identifying and representing causal relations in a network. It will be interesting to compare this method with methods using temporal correlations for identifying influence [125]. While this methodology has been mainly developed for the analysis of financial data, it was recently applied to the investigation of the immune system [126] and semantic networks [127]. Thus, in the future this methodology can be apllied to study different real-life systems, and to quantify the dependency and influence relationships between the nodes making up the network.

These two examples highlight the need to add new types of links in network science. Thus, future work must further investigate the issue of dependency and influence relationships between nodes in the network. Furthermore, a conceptual framework will be developed to investigate the issue of inhibition in networks, i.e. how one node or link affects the link (edge) between two (or more) other nodes. Understanding these different types of interactions between nodes and links will lead to new information on many aspects, such as flow of information in the network, its function, and its dynamics.

\subsection{Dynamical networks}

To understand the functions of the network, one must study its dynamical properties [128], and much work is needed in this direction. The dynamics of links and nodes can 
change completely the properties of the networks and thus, fundamental questions that have been extensively studied in static networks are still open for dynamical networks [28-30]. Such a methodology has recently been presented to study the dynamics in a given network [129]. By studying the dynamical properties of the network, one can uncover the underlying function of the network. It is quite reasonable to claim that the there is a strong link between the dynamics and the function in a given network. This can even be used to identify strategies of criminals and to anticipate their future actions [130]. Thus, to better understand the network function, it is crucial to study how network structures evolve in time, [27,131,132].

In many real networks, [30,102], links are generated, disappear and reappear over time. For example, in a mobile communication network the link exists only during the call. On a different time scale, friendships are formed and dissolved in social networks, links between websites are added and deleted: in epidemics spreading, recovery and re-infection can make a link infectious and immune repeatedly. Another important example of a realistic dynamical network is a climate network (see Sect. 5.2). In traffic communication via ad-hoc networks between vehicles the presence or absence of links changes very quickly, even on time scales comparable with those for information flow [103]. The analysis of co-evolution network dynamics, in which changes in the states of the nodes and the creation/destruction of links occur in comparable time scales, has many critical implications in the analysis in social collective phenomena $[9,134,135]$.

New tools are necessary to understand the relationship between the dynamics of the network, and its topology and function (for example temporal motifs [133]). In particular, when the system is in a critical state (i.e., at the edge of disintegration), addition or deletion of even a minute number of links can push the system into different phases, and as demonstrated by Iniguez, the time scales are relevant variables [29]. Initial observations have shown that the problem of temporal connectivity in a network can be directly mapped onto a directed percolation problem, where the direction represents the time: by having one replication of the network for each time step, and connecting node $i$ at time $t$ with node $j$ in time $t+1$ only if the edge $(i, j)$ exists at time $t$, and allowing only progress along the time axis, a path will emerge whenever there is a directed path in the equivalent percolation problem. Thus the knowledge of directed percolation could be used to understand dynamical networks, and vice versa. Since percolation is related to epidemics, and immunization is related to removing nodes, percolation approaches will be developed to study dynamical systems.

\subsection{Spatial networks}

When modeling the structure (topology) and function of complex networks, it is usually assumed that spatial constraints can be neglected so that high-dimensional mean-field approaches become applicable. This assumption is probably correct for some networks, like the World Wide Web (WWW) or email networks, where the geometrical length of the links is probably irrelevant. For a large fraction of real networks, however, the embedding in $2 d$ or $3 d$ space seems to matter and the geometrical distance between connected nodes plays an important role [36,37,104]. Examples are the Internet, airline networks, social networks, cellular-phone networks etc. In social networks, for example, the probability of having (and meeting) a friend that lives nearby is much higher than having and meeting one that lives far away. In airline networks, long-distance flights are rarer than short-distance flights. The same happens in cellular phone networks where short-distance calls are much more frequent than long-distance calls, even if the costs for short- and long-distant calls are the same. Regarding traffic communication via ad-hoc networks between vehicles, even 
an embedding in quasi- $1 d$ space is needed because roads are essentially $1 d$ (besides crossings) $[64,103]$.

The overall goal is to gain a better understanding of the effect of the embedding space on the topology and function of networks, where both the number of links emanating from each node and the distance between connected nodes follow certain distributions. Based on our recent preliminary results, it is expected that the spatial constraints will modify topology, robustness and function of the network. A major challenge is to find out how the laws of physics in the networks are changed due to the spatial constraints, and to develop efficient strategies that take them into account.

Once the spatial properties of real-world networks will be investigated, new approaches will be developed to generate model networks embedded in Euclidean space, and study their topological properties and robustness using scaling and percolation approaches. Furthermore, the functional properties of the constrained networks, including transport, epidemics and information spreading will be investigated. Thus, understanding the spatial properties of a single network will bring forth significant advances in network science. Progress in this regard will also significantly contribute to the other challenges discussed above - how spatial constraints influence the coupling between networks, network dynamics, and the dependency relationships between nodes.

\subsection{Transport in networks}

Only few studies have focussed on the relations between topology and transport properties [65]. The overall goal is to gain a better understanding of the behavior of transport in networks and of the interplay between topology and transport properties, such as capacity overloads, bottleneck structure and delay. The aim is to investigate the behavior of transport processes, such as epidemiological models, flow models and electrical (Kirchhoff) behavior in different network classes, such as spatial networks, scale free and P. Erdös, A. Rényi networks, and coupled network, and develop mechanisms for improving the transport in different network classes.

\subsection{Modular structure of networks}

Some of the structural properties that have been found to play a very important role recently have been the existence of communities, cliques, in networks, and the modular organization of networks. The modular structures or communities have been shown to be relevant in our current understanding of the structure and dynamics of complex systems. Detecting communities is of great importance in sociology, biology and computer science, disciplines where systems are often represented as graphs. This problem has been found to be difficult and not yet satisfactorily solved, despite the huge effort of a large interdisciplinary community of scientists working on it over the past few years [136], Communities should also be considered in the new frameworks of interdependent, time-dependent and multiplex networks [137].

As the world becomes more interconnected, the effect of groups and communities is becoming as important as that of individuals, and in many cases the interactions in the given network are of a higher order nature, which is a result of the interaction between the different communities in the network. Thus, it becomes crucial to characterize and quantify the interplay between individuals, communities, and the individualcommunity interactions in real world networks. 


\section{Applications}

In this section we list a few examples on how a new generation of network science will dramatically impact our daily and social life - including new insights, knowledge and applications on infrastructure, social systems, economics and climate.

\subsection{Infrastructure}

Today's infrastructure networks are not only characterized by fractal scaling laws [138], but have also become increasingly interdependent on one another. Diverse types of infrastructures such as water supply, transportation, fuel and power stations are coupled together. Owing to this coupling, interdependent networks are extremely sensitive to random failure, and in particular to targeted failure of a small fraction of nodes from one network can produce an iterative cascade of failures in several interdependent networks. Preliminary results have shown that networks that do not display any transition when considered separately, do display a sharp transition when interactions occurs between nodes that belong to two or more networks simultaneously. Electrical blackouts frequently result from a cascade of failures between interdependent networks, and the problem has been dramatically exemplified by the several large-scale blackouts that have occurred in recent years.

Blackouts are a demonstration of the important role played by the dependencies between networks. For example, the September 28, 2003 blackout in Italy resulted in a widespread failure of the railway network, healthcare systems, and financial services and, in addition, severely influenced communication networks (see box 6 of [25] for further examples). The partial failure of the communication system in turn further impaired the power grid management system, thus producing a negative feedback on the power grid (Fig. 2, left). This example emphasizes how inter-dependence can significantly magnify the damage in an interacting network system $[34,86]$.

Thus, understanding the coupling and interdependencies of networks will significantly affect and improve how infrastructures are designed and implemented, mainly towards more efficient and robust infrastructures. Many of the infrastructural networks are essential dynamic ones, where nodes and links are temporarily active. Moreover, the activities are usually extremely inhomogeneous, bursty (see, e.g., [31]. This means additional challenge for the capacities of the network and the optimal design has to take into account dynamic aspects.

\subsection{Climate}

Quantifying, understanding and predicting climate events are some of the most important challenges of human society. Specifically, climate records such as local temperature, pressure, pressure gradients and precipitation data obtained at certain stations and grid points can be investigated. The stations and/or grid points are the nodes in the corresponding climate networks. The links between the nodes are obtained from a cross correlation or synchronization analysis of the climate records at the nodes, and the links' strengths represent their degree of climate similarity. The climate networks allow to study the interrelationship between the different sites on the globe and thus represent the global behavior of the climate system. Preliminary results show that such networks are very sensitive to El Nino, even in areas where El Nino cannot be seen in temperature records [48]. Thus, the objective is to find out how the extreme events, such as El-Nino, influence the dynamics of the climate network, and whether changes in this dynamics can be used to identify new precursors and indicators of 


\section{How interdependent are infrastructures?}

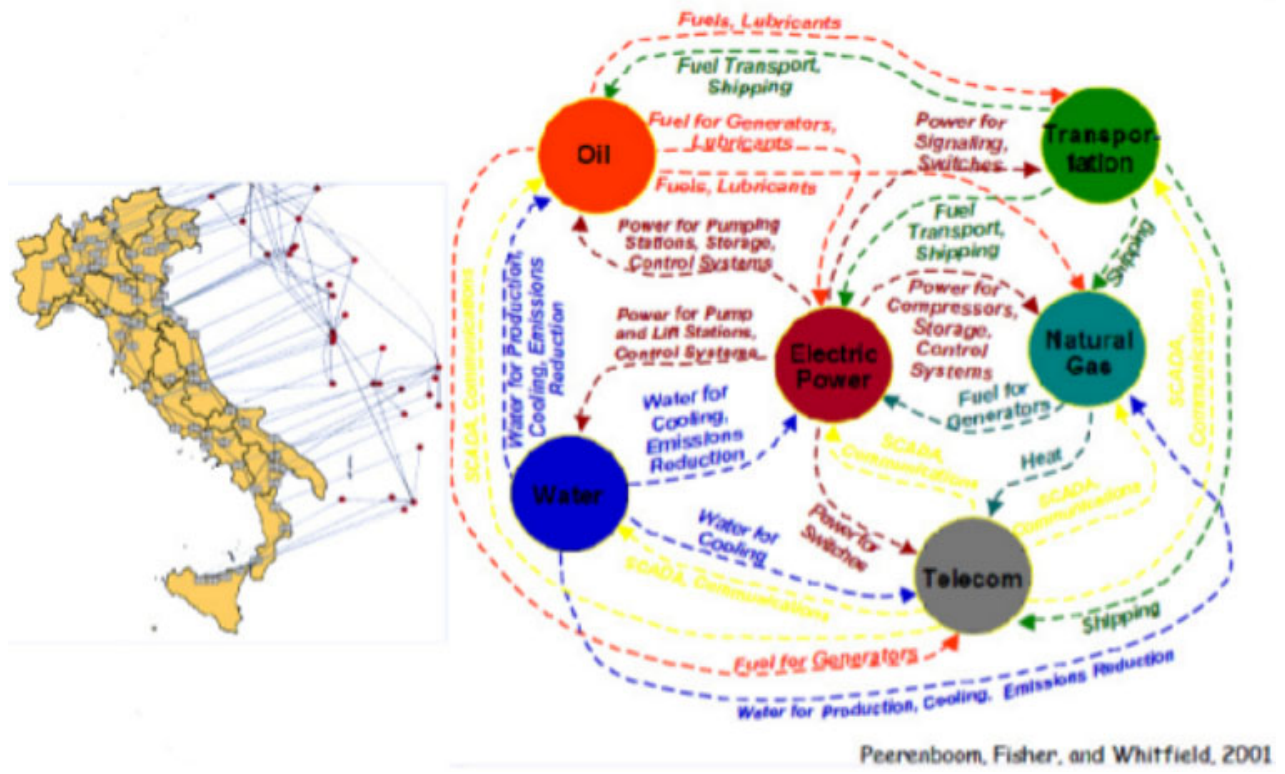

Fig. 2. Left: power grid and Internet dependence in Italy. Analysis of this system can explain the cascade failure that led to the 2003 blackout. Right: inter-dependence of fundamental infrastructures. A further example is a recent event in Cyprus (July 2011), where an explosion caused a failure of the electrical power lines, which in turn caused the countries water supply to shut down, due to the strong coupling between these two networks.

extremes. Furthermore, extreme events such as extreme droughts and heat waves, floods, storm floods, and hurricanes will be investigated.

One main focus will be to track the dynamics of the climate network. To obtain the time evolution of the network, we aim to determine the link values for a certain period of time (ranging from one month to one year) and follow the time evolution of the adjacency matrices that represent the network. Furthermore, the similarities between networks representing different fields will be investigated, e.g. temperature network or pressure network, and how to retrieve and characterize interacting dynamic structures $[109,110]$.

An equally important objective is to identify finger print of extreme events in the climate networks, and will study the dynamics of the climate networks in regimes where extreme events occurred, for example, extreme storm floods or hurricanes. This can be achieved by studying changes in the network structure, and testing the effect of the extreme events on the network structure. Regimes and the fields for which the effect is maximal, will be referred to as optimal networks. Furthermore, the effect of the extreme events on the similarity between networks of different fields will be studied.

\subsection{Social networks}

Mobile phones have become an indispensable part of our life. They are widely used in our modern world for all aspects of social life. Our daily activity has become dependent on these phones, especially since the introduction of the smart phones, which 


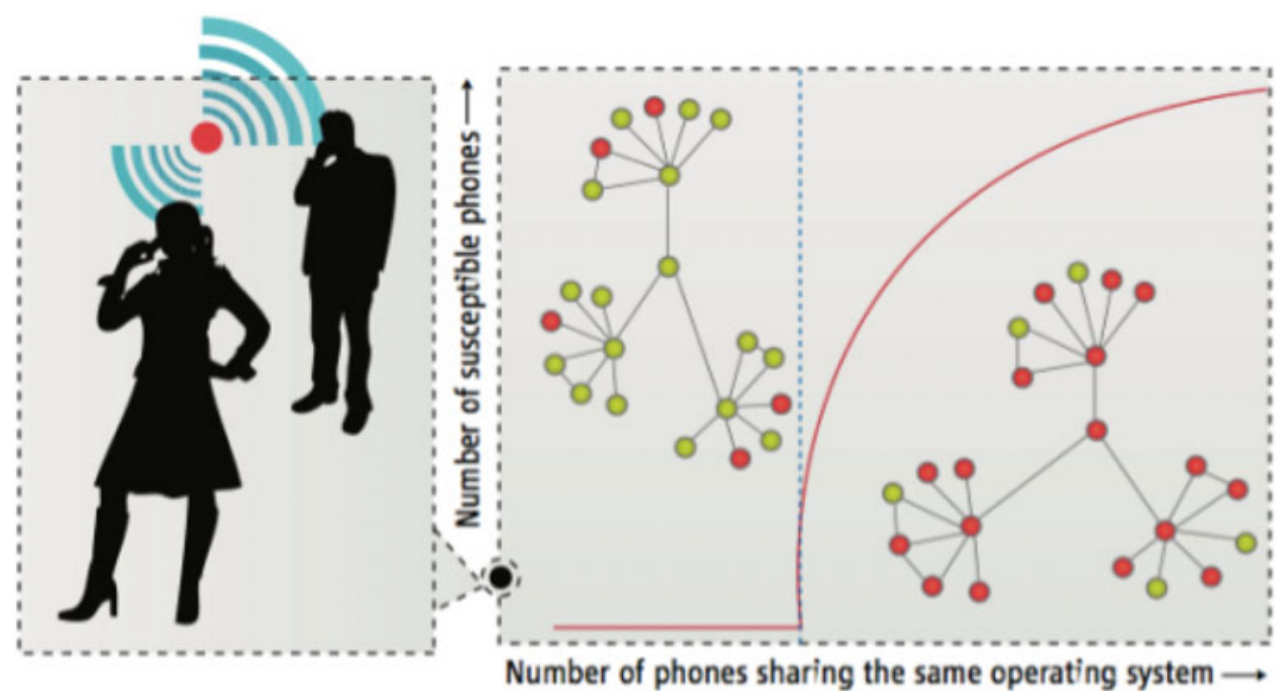

Fig. 3. Epidemics to come. Currently, very few smartphones are susceptible to a virus (red circles; non-susceptible phones, green circles), a number that is below the threshold of transition to an epidemic (vertical blue line). In future epidemics, as the number of smartphones increases, the market share of a single operating system will reach a critical threshold and facilitate virus spread, potentially leading to a pandemic (reproduction from [40]).

are becoming smarter and smarter. Mobile phone data has already been recently used to successfully prove a thirty year old hypothesis by Granovetter about the strength of weak ties [22], revealing an important structural aspect of the human society [23]. Time stamped data allow for a deep insight into the mechanisms of information spreading in the communication network [31]. Such data, together with demographic metadata about gender, age and ZIP code enable to dissolve the heterogeneity of the population, identify typical behavioral patterns, and contribute this way to many practical applications from innovation promotion to combating crime.

Why, despite the more than 400 mobile phone viruses documented by cybersecurity organizations, is there no apparent serious concern about the prospect of viruses infecting mobile phones? By combining network science and percolation theory, Wang et al. [39] examined mobility and communications data on the activity of more than six million individuals to study the speed and breadth of potential mobile phone virus outbreaks. Their conclusion is rather unexpected: Although mobile phone viruses do not pose a threat of spreading right now, the increasing market share of smart- phones - mobile phones with computer-like operating systems and advanced features (such as electronic mail and Internet access) - will soon reach a phase transition point, beyond which mobile viruses could become far more damaging and widespread than current computer viruses (see Fig. 3). Applying network theory, we will develop efficient immunization strategies, which will consider the topology and dynamics, to avoid the expected catastrophic spreading of mobile phone viruses in the system. One such type of strategy is to divide, via immunization, the network into equal sub-networks. This strategy guaranties the minimum number of units needed to be immunized.

Other examples where we need new network theory tools include the issue of opinion formation [21] and identification of influential members in the network [45]. The 
establishment of criteria characterizing the hierarchical place of nodes within networks is one of the objectives that have to be pursued in order to make network theory a tool for organizing the nested, multi-level structure of real world organization and functionality [139]. New insights in these issues will have many applications ranging from understanding social crises to efficient spreading of information and marketing.

In 2006, Microsoft studied billions of electronic messages to work out that any two strangers are, on average, only separated by 6.6 degrees of separation. Researchers studied over 30 billion conversations from the Microsoft Messenger instant messaging network for June 2006, which equated to half of the world's total instant-messaging traffic sent during that month, and produced results mirroring Milgram's original work, 'Six degrees of separation'. Since then, the exponentially growing popularity of social networks such as Facebook and Linkedin have been reducing this degree of separation constantly. As the world is becoming more connected, it is crucial to fully understand the topology, dynamics and functions of the social networks we are part of. We have to understand mechanisms how communication is created among few participants as well as within large communities [117].

\subsection{Economics}

The latest financial crisis has emphasized more then ever the importance of collective connectivity effects in the evaluation of financial fragility and for the probability of default. While traditional evaluations of the probability of default use only global information (general situation of the economic branch to which a company belongs) and point information (companies' balance sheet, profit margins, etc.), the latest events show that effects can and do propagate over many intermediate connections. In fact, the cascading effect has been a crucial element of the fast and devastating impact of the crisis. Thus, it is not realistic to separate the stability of a company from the collective dynamics taking place in its economic neighborhood. Access to time and conditions of capital flows between companies allows one in principle a capability to monitor in detail the working of the economy. One example is to express the fragility of a node in terms of the probabilities of default of its clients and suppliers. By conducting experiments on the network of financial transfers, one can probe the probability and size of such cascading events. We plan to use these theoretical methods in coordination with the bank policy makers and national bank officials in order to bring as soon as possible our diagnostic capabilities at the level at which they can be useful for the banks' needs: client solvability evaluation, planning of loans and borrowing, etc.

Financial systems are perhaps the best example of a complex adaptive system, in which the micro interacts through bottom-up mechanisms with the macro. This is followed by top-down feedback between the macro and the micro. One immediate example is a market index and the stocks that makeup this index. The stocks represent the micro, while the index represents the macro. Kenett et al. [118,120? ] have recently shown that the index has a stronger influence on the stocks then vice versa, which is neither a trivial or intuitive result. Thus, the micro and the macro continuously interact. These interactions are best characterized by network science, one which addresses dynamic and coupled networks. Recent work on individual strategies as subtracted from detailed financial data [140,141] enables to identify groups of players on the market and their role in stabilization and destabilization. One of the main sources of the intrinsic instability of financial markets is the almost entire absence of negative feedback loops. Appropriate network models with signed (positive and negative) links will help in finding the optimal balance between stability and liquidity and contribute this way to the solution of the major problem of market regulation. 
The next generation of network science will allow new understanding of how economic and financial systems organize and interact. This is crucial for the prevention of financial crises, and to ensure trust and fair trade to all the investing public. These new tools will give birth to new economic models and understandings, which will strengthen the world economics.

\subsection{Resilience of networks}

Our dependence on networks - be they infrastructure, economic, social or others leave us prone to crises caused by the vulnerabilities of these networks. There is a great need to develop new methods to protect infrastructure networks and prevent cascade of failures (especially in cases of coupled networks, see for example [41,83, 84]). Terrorist attacks on transportation networks have traumatized modern societies. With a single blast, it has become possible to paralyze airline traffic, electric power supply, ground transportation or Internet communication. How and at which cost can one restructure the network such that it will become more robust against malicious attacks.

The gradual increase in attacks on the networks society depends on - Internet, mobile phone, transportation, air travel, banking, etc. - emphasize the need to develop new strategies to protect and defend these crucial networks of communication and infrastructure networks. One example is the threat of liquid explosives a few years ago, which completely shut down air travel for days, and has created extreme changes in regulations. Such threats and dangers warrant the need for new tools and strategies to defend against.

\subsection{Urban dynamics and planning}

Wednesday, May 23, 2007 marks a major shift in society: for the first time in human history more people live in cities than in rural areas; and the process is still advancing [142]. Beyond the statistical-demographic anecdote, this fact is significant as it implies that every social issue, ranging from globalization, multiculturalism, social unrest, to environmental pollution, extreme event, flood and tsunami, is intimately related to the specific structure and dynamics of cities and urbanism (see for example [143]). More specifically, it is related to the fact that cities are not just containers where people live and act, but they are an active force in shaping human behavior and society [144]. It is in this respect that the 21st century is described as the urban century.

The last thirty years have witnessed the emergence of complexity theories of cities - a domain of research that applies the various complexity theories to the study of cities [145-147]. As in other domains, in the last decade, a growing number of studies approach cities and their dynamics as complex networks. These studies have demonstrated a whole set of resemblances between cities, on the one hand, and natural, material and organic networks, on the other. Yet, they have also exposed important differences that are a consequence of, firstly, the fact that cities are large-scale collective artifacts and secondly, that cites are dual-complex systems, that is, the city as a whole is a complex system/network and each of its parts (the urban agents) is a complex system/network by itself. From here follows a challenge to describe and study cities and systems of cities as coupled multi-level complex networks.

Cities have always been related to planning. However, it is only recently that students of complexity theories of cities started to explore the implications of the complexity of cities to urban planning. Three research directions can be identified 
here: one that is using urban simulation models (mainly cellular automata, agent based and network models) as predictive/evaluation tools in the context of planning support systems; for example, evaluating the impact of a new road or bridge on the entire transportation network and on adjacent networks (e.g. the urban landuse network). Two, viewing the city as a complex network in which every urban agent (a single household, a firm or the city's planning department) is a planner at a certain scale, and, that due to non-linearities, the action of a single agent/planner (e.g. household) might influence the city more than that of a large-scale agent (e.g. planning department). Three, re-structuring the very planning process as a complex self-organized network and system. The above research directions complement each other and the challenge here is to simulate the coupling between the various urban networks.

\section{Innovation, expected paradigm shifts, and impact}

Systems have mainly been considered isolated without relevant coupling with related ones. However, taking the transport infrastructures as an example, it is known that when the air transports are affected by an active volcano, the terrestrial ones become overloaded. The current challenges in network theory are the need to develop a new framework to deal with such coupled and interdependent systems, where not only the structural properties are considered but details from the dynamic processes taking place in the network are also taken into account. This will only be achieved through data-driven models.

The employment of ideas and techniques from complex network theory and the proposed theory of coupled and interdependent networks to understand and quantify the role of connections and dependencies within a system and between different ones opens the possibility to manage the complexity, optimize the systems and reduce their vulnerability to failures. At the technological level, such understanding will help in developing smart infrastructures that are able to predict and adjust to different conditions and able to respond successfully in real time to abnormal load shedding, thus avoiding, for example, blackouts, traffic jams, or inefficiencies and shortages in the supply of oil and gas. In climate, understanding climate network dynamics will help to be able to forecast extreme events such as El-Nino. In finance and economics, being aware of the underlying topology between companies, banks, countries, and shareholders, might improve competitiveness, by decreasing the investments volatility and mitigating systemic risk. The commercial and industrial systems strongly require an efficient and resilient logistic network to avoid excessive inventories and the lack of robustness against cyclic perturbations, which affect the production costs and, consequently, the competitiveness. In social systems, the new network science will enable early identification of social crises, and provide methods to mitigate social catastrophes.

The present age is one where networks dominate every aspect of life. The world has become a global village, and this village is becoming smaller and smaller, with the continuous introduction of ways to interact and connect to other people. Thus, the next generation of network science will play a crucial role in the design, organization and maintenance of our future socio-techno-economic world.

The publication of this work was partially supported by the European Union's Seventh Framework Programme (FP7/2007-2013) under grant agreement no. 284709, a Coordination and Support Action in the Information and Communication Technologies activity area ('FuturICT' FET Flagship Pilot Project). 


\section{References}

1. S. Bishop, Foreword, Eur. Phys. J. Special Topics 214, 1 (2012)

2. D. Helbing, Introduction, Eur. Phys. J. Special Topics 214, 5 (2012)

3. D. Helbing, S. Bishop, R. Conte, P. Lukowicz, J.B. McCarthy, Eur. Phys. J. Special Topics 214, 11 (2012)

4. A. Carbone, M. Ajmone-Marsan, K. Axhausen, M. Batty, M. Masera, E. Rome, D. Helbing, Eur. Phys. J. Special Topics 214, 435 (2012)

5. S. Bishop, D. Helbing, P. Lukowicz, R. Conte, Proc. Comp. Sci. 7, 34 (2011)

6. D. Helbing, S. Balietti, Eur. Phys. J. Special Topics 195, 3 (2011)

7. D. Helbing, S. Balietti, S. Bishop, P. Lukowicz, Eur. Phys. J. Special Topics 195, 165 (2011)

8. M.E.J. Newman, A.-L. Barabási, D.J. Watts, The structure and dynamics of networks (Princeton University Press, Princeton, NJ, 2006)

9. S. Boccaletti, V. Latora, Y. Moreno, M. Chavez, D.U. Hwang, Phys. Rep. 424, 175 (2006)

10. M.E.J. Newman, Networks: An introduction (Oxford University Press, Oxford, 2010)

11. R. Cohen, S. Havlin, Complex networks: structure, robustness and function (Cambridge Univ. Press, 2010)

12. F. Liljeros, C.R. Edling, L.A.N. Amaral, H.E. Stanley, Y. Aberg, Nature 411, 6840 (2001)

13. M. Faloutsos, P. Faloutsos, C. Faloutsos, SIGCOMM Comput. Commun. Rev. 29, 251 (1999)

14. A.-L. Barabási, R. Albert, Science 286, 509 (1999)

15. R. Pastor-Satorras, A. Vespignani, Phys. Rev. Lett. 86, 3200 (2001)

16. R. Cohen, S. Havlin, D. Ben-Avraham, Phys. Rev. Lett. 91, 247901 (2003)

17. F. Radicchi, S. Fortunato, C. Castellano, Proc. Natl. Acad. Sci. USA 105, 17268 (2008)

18. G. Bonanno, G. Caldarelli, F. Lillo, S. Micciché, N. Vandewalle, R.N. Mantegna, Eur. Phys. J. B 38, 363 (2003)

19. S. Solomon, G. Weisbuch, L. de Arcangelis, N. Jan, D. Stauffer, Physica A 277, 239 (2000)

20. S. Solomon, E. Shir, S. Kirkpatrik, in Proceedings of AISB03 Adaptive Agents and Multi-Agent Systems, edited by D. Kazakov, E. Alonso, D. Kudenko (Aberystwyth, 2004)

21. J. Shao, S. Havlin, H.E. Stanley, Phys. Rev. Lett. 103, 018701 (2009)

22. M. Granovetter, Amer. J. Sociol. 78, 1360 (1973)

23. J.-P. Onnela, J. Saramäki, J. Hyvonen, G. Szabó, D. Lazer, K. Kaski, J. Kertész, A.-L. Barabási, Proc. Natl. Acad. Sci. USA 104, 7332 (2007)

24. S. Cantono, S. Solomon, New J. Phys. 12, 075038 (2010)

25. Introductory chapter of this issue, Eur. Phys. J. Special Topics 214 (2012)

26. R. Itzhak, L. Muchnik, T. Erez, S. Solomon, J. Goldenberg, Y. Louzoun, Physica A 389, 5308 (2010)

27. N. Fujiwara, J. Kurths, A. Díaz-Guilera, Phys. Rev. E 83, 025101 (2011)

28. C. Nardini, B. Kozma, A. Barrat, Phys. Rev. Lett. 100, 158701 (2008)

29. G. Iniguez, J. Kertész, K. Kaski, R.A. Barrio, Phys. Rev. E 80, 066119 (2009)

30. R. Parshani, M. Dickison, R. Cohen, H.E. Stanley, S. Havlin, EPL 90, 38004 (2010)

31. M. Karsai, M. Kivelä, R.K. Pan, K. Kaski, J. Kertész, A.-L. Barabási, J. Saramäki, Phys. Rev. E 83, 025102(R) (2011)

32. A.-L. Barabási, Nature 435, 207 (2005)

33. M. San Miguel, J.H. Johnson, J. Kertesz, K. Kaski, A. Diaz-Guilera, R. S. MacKay, V. Loreto, P. Erdi, D. Helbing, Eur. Phys. J. Special Topics 214, 245 (2012)

34. S.V. Buldyrev, R. Parshani, G. Paul, H.E. Stanley, S. Havlin, Nature 464, 08932 (2010)

35. D.J. Watts, S.H. Strogatz, Nature 393, 440 (1998)

36. K. Kosmidis, S. Havlin, A. Bunde, EPL 82, 48005 (2008)

37. L. Daqing, K. Kosmidis, A. Bunde, S. Havlin, Nature Phys. 7, 481 (2011)

38. G. Krings, F. Calabrese, C. Ratti, V.D. Blondel, J. Stat. Mech. L07003 (2009) 
39. P. Wang, M.C. González, C.A. Hidalgo, A.L. Barabási, Science 324, 1071 (2009)

40. S. Havlin, Science 324, 1023 (2009)

41. C.M. Schneider, T. Mihaljev, S. Havlin, H.J. Herrmann, [arXiv:1102.1929] (2011)

42. C.M. Schneider, A.A. Moreira, J.S. Andrade Jr., S. Havlin, H.J. Herrmann, Proc. Natl. Acad. Sci. USA 108, 3838 (2011)

43. A. Garas, P. Argyrakis, C. Rozenblat, M. Tomassini, S. Havlin, New J. Phys. 12, 113043 (2010)

44. D.Y. Kenett, M. Raddant, T. Lux, E. Ben-Jacob, PLoS ONE 7, e31144 (2012)

45. M. Kitsak, L.K. Gallos, S. Havlin, F. Liljeros, L. Muchnik, H.E. Stanley, H.A. Makse, Nature Phys. 6, 888 (2010)

46. S. Lämmer, D. Helbing, J. Stat. Mech.-Theory E. 2008, 04019 (2008)

47. G. Li, S.D.S. Reis, A.A. Moreira, S. Havlin, H.E. Stanley, J.S. Andrade Jr., Phys. Rev. Lett. 104, 018701 (2010)

48. A. Gozolchiani, K. Yamasaki, S. Havlin, [arXiv:1010.2605] (in print) (2011)

49. R. Albert, H. Jeong, A.L. Barabasi, Nature 406, 378 (2000)

50. R. Cohen, K. Erez, D. Ben-Avraham, S. Havlin, Phys. Rev. Lett. 85, 4626 (2000)

51. R. Albert, A.-L. Barabási, Rev. Mod. Phys. 74, 47 (2002)

52. R. Pastor-Satorras, A. Vespignani, Evolution and structure of the Internet: A statistical physics approach (Cambridge University Press, Cambridge, 2006)

53. S.N. Dorogovtsev, J.F.F. Mendes, Evolution of networks: From biological nets to the Internet and $W W W$ (Oxford University Press, New York, 2003)

54. M.E.J. Newman, SIAM Rev. 45, 167 (2003)

55. G. Caldarelli, Scale-free networks (Oxford University Press, Oxford, 2007)

56. G. Caldarelli, A. Vespignani, Large scale structure and dynamics of complex networks: From information technology to finance and natural science (World Scientific, Singapore, 2007)

57. A. Barrat, M. Barthélemy, A. Vespignani, Dynamical processes on complex networks (Cambridge Univ. Press, 2008)

58. D. Helbing, D. Armbruster, A. Mikhailov, E. Lefeber, Physica A 363, 1 (2006)

59. D. Helbing, J. Siegmeier, S. Lämmer, Networks Heterogeneous Media 2, 193 (2007)

60. T. Becker, M.E. Beber, K. Windt, M-T. Hütt, D. Helbing, J. Stat. Mech. P05004 (2011)

61. S. Carmi, Z. Wu, E. Lopez, S. Havlin, H.E. Stanley, Eur. Phys. J. B 57, 165 (2007)

62. E. Lopez, S. Carmi, S. Havlin, S.V. Buldyrev, H.E. Stanley, Physica D 224, 69 (2006)

63. R. Kinney, P. Crucitti, R. Albert, V. Latora, Eur. Phys. J. B 46, 101 (2005)

64. A. Kesting, M. Treiber, D. Helbing, IEEE Intell. Transportation Syst. 11, 172 (2010)

65. R. Sipahi, S. Lämmer, D. Helbing, J. Dynamic Systems, Meas. Control 131, 021005 (2009)

66. R. Cohen, K. Erez, D. Ben-Avraham, S. Havlin, Phys. Rev. Lett. 86, 3682 (2001)

67. D.S. Callaway, M.E.J. Newman, S.H. Strogatz, D.J. Watts, Phys. Rev. Lett. 85, 5468 (2000)

68. Y. Chen, G. Paul, S. Havlin, F. Liljeros, H.E. Stanley, Phys. Rev. Lett. 101, 058701 (2008)

69. A.E. Motter, Y.C. Lai, Phys. Rev. E 66, 065102 (2002)

70. D. Helbing, C. Kühnert, Physica A 328, 584 (2003)

71. A.E. Motter, Phys. Rev. Lett. 93, 098701 (2004)

72. L. Buzna, K. Peters, D. Helbing, Physica A 363, 132 (2006)

73. L. Buzna, K. Peters, H. Ammoser, C. Kühnert, D. Helbing, Phys. Rev. E 75, 056107 (2007)

74. K. Peters, L. Buzna, D. Helbing, Int. J. Crit. Infrastructures 4, 46 (2008)

75. I. Simonsen, L. Buzna, K. Peters, S. Bornholdt, D. Helbing, Phys. Rev. Lett. 100, $218701(2008)$

76. D. Balcan, V. Colizza, B. Goncalves, H. Hu, J.J. Ramasco, A. Vespignani, Proc. Natl. Acad. Sci. USA 106, 21484 (2009)

77. H. Hooyberghs, B. van Schaeybroeck, A.A. Moreira, J.S. Andrade Jr., H.J. Herrmann, J.O. Indekeu, Phys. Rev. E 81, 011102 (2010) 
78. C.M. Schneider, R.F.S. Andrade, T. Shinbrot, H.J. Herrmann, EPL 95, 16001 (2011)

79. C.M. Schneider, L. de Arcangelis, H.J. Herrmann, EPL 95, 16005 (2011)

80. D. Helbing, H. Ammoser, C. Kühnert, Physica A 363, 141 (2006)

81. A. Moreira, J.S. Andrade Jr., H.J. Herrmann, J.O. Indekeu, Phys. Rev. Lett. 102, $018701(2009)$

82. A. Gulfraind, PLoS one 5, e13448 (2010)

83. J. Shao, S.V. Buldyrev, S. Havlin, H.E. Stanley, Phys. Rev. E 83, 036116 (2011)

84. H.J. Herrmann, C.M. Schneider, A.A. Moreira, J.S. Andrade Jr., S. Havlin, J. Stat. Mech. P01027 (2011)

85. A. Vespignani, Nature 464, 984 (2010)

86. V. Rosato, L. Issacharoff, F. Tiriticco, S.S. Meloni, S.D. Porcellinis, R. Setola, Int. J. Critical Infrastructures 4, 63 (2008)

87. F. Schweitzer, G. Fagiolo, D. Sornette, F. Vega-Redondo, A. Vespignani, D.R. White, Science 325, 422 (2009)

88. R. Parshani, S.V. Buldyrev, S. Havlin, Phys. Rev. Lett. 105, 048701 (2010)

89. P. Erdös, A. Rényi, Publ. Math. Debrecen 6, 290 (1959)

90. P. Erdös, A. Rényi, Publ. Math. Inst. Hung. Acad. Sci. 5, 17 (1960)

91. J. Chalupa, P.L. Leath, G.R. Reich, J. Phys. C 12, L31 (1979)

92. B. Bollobás, Random graphs (Academic, 1985)

93. A. Bunde, S. Havlin, Fractals and disordered systems (Springer, Berlin, 1996)

94. C. Moukarzel, P.M. Duxbury, P.L. Leath, Phys. Rev. E 55, 5800 (1997)

95. M.L. Sachtjen, B.A. Carreras, V.E. Lynch, Phys. Rev. E 61, 4877 (2000)

96. D.J. Watts, Proc. Natl. Acad. Sci. USA 99, 5766 (2002)

97. Y. Moreno, R. Pastor-Satorras, A. Vazquez, A. Vespignani, EPL 62, 292 (2003)

98. R. Parshani, S.V. Buldyrev, S. Havlin, Proc. Natl. Acad. Sci. USA 108, 1007 (2011)

99. R. Parshani, C. Rozenblat, D. Ietri, C. Ducruet, S. Havlin, EPL 92, 68002 (2010)

100. X.Q. Huang, J.X. Gao, S.V. Buldyrev, S. Havlin, H.E. Stanley, Phys. Rev. E 83, 065101 (2011)

101. C.M. Schneider, N.A.M. Araújo, S. Havlin, H.J. Herrmann [arXiv: 1106. 3234] (2011)

102. P. Holme, J. Saramaki [arXiv:1108.1780] (2011)

103. M. Schönhoff, A. Kesting, M. Treiber, D. Helbing, Physica A 363, 73 (2006)

104. M. Barthélemy, Phys. Rep. 499, 1 (2011)

105. A.A. Tsonis, K.L. Swanson, P.J. Roebber, Bull. Am. Meteorol. Soc. 87, 585 (2006)

106. A.A. Tsonis, G. Wang, K.L. Swanson, F.A. Rodrigues, L.D. Costa, Clim. Dyn. 37, 933 (2011)

107. A. Gozolchiani, K. Yamasaki, O. Gazit, S. Havlin, EPL 83, 28005 (2008)

108. K. Yamasaki, A. Gozolchiani, S. Havlin, Phys. Rev. Lett. 100, 228501 (2008)

109. J.F. Donges, Y. Zou, N. Marwan, J. Kurths, EPL 87, 48007 (2009)

110. J.F. Donges, H.C.H, Schultz, N. Marwan, Y. Zou, J. Kurths, Eur. Phys. J. B 84, 635 (2011)

111. N. Malik, B. Bookhagen, N. Marwan, J. Kurths, Clim. Dyn. 39, 971 (2012)

112. M.E.J. Newman, Proc. Natl. Acad. Sci. USA 98, 404 (2001)

113. M. Girvan, M.E.J. Newman, Proc. Natl. Acad. Sci. USA 99, 7821 (2002)

114. G. Palla, I. Derenyi, I. Farkas, T. Vicsek, Nature 435, 814 (2005)

115. G. Kossinets, D. Watts, Science 311, 88 (2006)

116. Webpage http://empcommission.org/docs/A2473-EMP_Commission-7MB.pdf (retrieved 2011)

117. Y. Wu, C. Zhou, J. Xiao, J. Kurths, H.J. Schellnhuber, Proc. Natl. Acad. Sci. USA 107, $42(2010)$

118. D.Y. Kenett, M. Tumminello, A. Madi, G. Gur-Gershgoren, R.N. Mantegna, E. BenJacob, PLoS one 5, e15032 (2010)

119. Y. Shapira, D.Y. Kenett, E. Ben-Jacob, Eur. Phys. J. B 72, 659 (2009)

120. D.Y. Kenett, Y. Shapira, A. Madi, S. Bransburg-Zabary, G. Gur-Gershgoren, E. BenJacob, PLoS one 6, e19378 (2011) 
121. D.Y. Kenett, Y. Shapira, G. Gur-Gershgoren, E. Ben-Jacob, J. Eng. Tech. Sci. Rev. 4, $218(2012)$

122. D.Y. Kenett, T. Preis, G. Gur-Gershgoren, E. Ben-Jacob, Int. J. Bifurcat. Chaos 22, $1250181(2012)$

123. D.Y. Kenett, Y. Shapira, G. Gur-Gershgoren, E. Ben-Jacob, J. Eng. Sci. Tech. Rev. 4, $218(2012)$

124. D.Y. Kenett, M. Raddant, L. Zatlavi, T. Lux, E. Ben-Jacob, Int. J. Mod. Phys. Conf. Ser. 16, 13 (2012)

125. B. Tóth, J. Kertész, Physica A 360, 505 (2006)

126. A. Madi, D.Y. Kenett, S. Bransburg-Zabary, Y. Merbl, F.J. Quintana, S. Boccaletti, A.I. Tauber, R. Cohen, E. Ben-Jacob, Chaos 21, 016109 (2011)

127. Y.N. Kenett, D.Y. Kenett, E. Ben-Jacob, M. Faust, PLoS one 6, e23912 (2011)

128. A. Arenas, A. Díaz-Guilera, J. Kurths, Y. Moreno, C. Zhou, Phys. Rep. 469, 93 (2008)

129. I. Sendina-Nadal, Y. Ofran, J.A. Almendral, J.M. Buldu, I. Leyva, D.Q. Li, S. Havlin, S. Boccaletti, PLoS one 6, e17679 (2011)

130. C. Qian, Y.B. Wang, J.D. Cao, J.Q. Lu, J. Kurths, EPL 93, 68006 (2011)

131. G. Zamora-López, C. Zhou, J. Kurths, Front. Neurosci. 5, 83 (2011)

132. L. Zemanova, G. Zamora, C. Hilgetag, J. Kurths, Phys. Rev. Lett. 97, 238103 (2006)

133. L. Kovanen, M. Karsai, K. Kaski, J. Kertész, J. Saramaki [arXiv 1107.5646] (2011)

134. M.G. Zimmermann, V.M. Eguluz, M. San Miguel, Phys. Rev. E 69, 065102 (2004)

135. T. Gross, B. Blasius, J. R. Soc. Interface 5, 259 (2008)

136. S. Fortunato, Physics Reports 486, 75 (2010)

137. P.J. Mucha, T. Richardson, K. Macon, M.A. Porter, J.P. Onnela, Science 328, 876 (2010)

138. C. Kühnert, D. Helbing, G.B. West, Physica A 363, 96 (2006)

139. Y. Louzoun, L. Muchnick, S. Solomon, Bioinformatics 22, 581 (2006)

140. F. Lillo, E. Moro, G. Vaglica, R.N. Mantegna, New J. Phys. 10, 043019 (2008)

141. G.-H. Mu, W.-X. Zhou, W. Chen, J. Kertész, New J. Phys. 12, 075037 (2010)

142. R. Wimberley, L. Morris, G. Fulkerson, The Rural Sociologist 27, 42 (2007)

143. D.Y. Kenett, J. Portugali, Proc. Natl. Acad. Sci. USA 109, 11472 (2012)

144. J. Portugali, Complexity, Cognition and the City (Springer 2011)

145. M. Batty, Cities and Complexity: Understanding Cities with Cellular Automata, AgentBased Models, and Fractals (MIT Press 2007)

146. M. Batty, Science 319, 769 (2008)

147. M. Batty, Building Res. Information 38, 123 (2010) 